\title{
黄土区草本植被根系与土壤垂直侵蚀产沙关系研究
}

\author{
李 鹏1,2 李占斌 ${ }^{1,3}$ 鲁克新 ${ }^{1}$ \\ （1 西安理工大学水利水电学院,西安 710048）（2 西北农林科技大学林学院,陕西杨凌 712100） \\ (3 中国科学院水利部水土保持研究所, 陕西杨凌 712100)
}

\begin{abstract}
摘 要 为了系统揭示植被根系对径流侵蚀产沙的影响, 采用土钻法对草地植被根系分布特征进行系统调查, 采 用分层冲刷的方法对黄土高原草地土壤不同坡度、不同流量条件下的侵蚀产沙特征进行了研究。结果表明不同立 地上的植被根系都表现出了随着土层深度增加而减少的趋势。分层冲刷的试验结果表明在土壤表层, 植被根系对 侵蚀产沙的影响是占主导地位的; 而当土层超过一定深度后, 根系的分布数量减少, 不同流量和坡度下的深层土壤 侵蚀产沙量明显增加, 根系提高土壤抵抗径流侵蚀产沙的能力受到了限制。同时随着土层深度的不断加大, 坡面 上径流侵蚀的形态也在发生变化, 逐渐从面蚀向细沟侵蚀发展。结合对草地植被根系生物量垂直分布特征的研 究, 证明土壤侵蚀产沙的这种变化是与草本植被根系的分布特征密切相关的。通过进一步分析植被根系分布特征 和土壤垂直侵蚀产沙之间的联系, 建立了草地植被根系生物量与土壤垂直侵蚀产沙特征之间的定量关系。
\end{abstract}

关键词 分层冲刷 垂直侵蚀产沙 根系 黄土区

\section{RELATIONSHIP BETWEEN HERBACEOUS ROOT SYSTEM AND VERTICAL SOIL SEDIMENT YIELD IN LOESS AREA}

\author{
LI Peng ${ }^{1,2}$ LI Zhan-Bin ${ }^{1,3}$ and LU Ke-Xin ${ }^{1}$ \\ (1 Institute of water resource and hydro-electric engineering, Xi' an University of Technology, Xi' an, Shaanxi 710048, China) \\ (2 College of Forestry, Northwest Sci-Technological University of Agriculture and Forestry, Yangling, Shaanxi 712100, China) \\ (3 Institute of Soil and Water Conservation, Chinese Academy of Sciences, Ministry of Water Resources, Yangling, Shaanxi 712100, China)
}

\begin{abstract}
Current knowledge about root morphology and its impacts on soil erosion is limited and, therefore, detailed analyses of root system in controlling soil erosion are needed. In order to determine the effects of root system on soil erosion quantitatively, root investigation and stratified runoff scouring were conducted in field. The distribution of vertical root biomass on three sites decreased with the increase of soil depth in a similar manner. Most roots were concentrated in surface soil, and decreased to less than $0.2 \mathrm{~kg}^{\bullet} \mathrm{m}^{-3}$ in soil below the depth of $40 \mathrm{~cm}$. There was no significant difference in root biomass on the same soil depth from different points. Results from stratified runoff scouring on different soil depths in the field indicated that, on the surface soil layer, the existence of root could effectively improve soil resistance to runoff erosion, and its effect on sediment yield was dominant. While in deeper soil, sediment yield increased with the decrease of root distribution, and was more related to slope gradient, runoff discharge and soil properties. In addition, with the progress of runoff scouring, the form of soil erosion changed from sheet erosion to rill erosion. Investigation of the root distribution patterns revealed that the vertical sediment yield was closely related to root distribution characteristics. Further analysis of the root distribution pattern and the vertical sediment yield allowed establishment of a quantitative relationship between root biomass and vertical sediment yield.
\end{abstract}

Key words Root, Soil depth, Soil erosion and sediment yield, Loess Plateau

长期以来, 黄土高原地区土壤抵抗侵蚀能力一 直是研究的重点问题(朱显谟和田积䒯, 1993; 李勇, 1995; 刘国涁, 1997)。朱显谟和田积芗 (1993)等人 对黄土高原地区土壤渗透性和抗冲性进行研究后认 为黄土区土壤的渗透性强和土壤抗冲性弱的特征与
黄土的淀积方式有关, 黄土堆积以后由于植被的生 长而获得提高和加强。植被根系提高土壤抵抗径流 侵蚀的能力在许多研究中得到了证实 (李勇等, 1991; 李勇, 1995; 刘国涁等, 1996; 刘国涁, 1997; Ghidey, 1997; Gyssels \& Poesen, 2003)。李勇等( 1991) 
和李勇 (1995)通过对黄土高原地区乔灌植被根系与 土壤物理性质关系的研究, 证实乔灌植被的细根 $(\varphi$ $<1 \mathrm{~mm}$ )对土壤结构的改善作用最大, 其稳定土层 结构的范围与细根剖面中的分布特征一致。由于植 被的存在, 改变了土壤的理化性质, 使得土壤容重减 小, 孔隙度增加, 团聚体含量加强（Waldron \& Dakessian, 1981; Reid \& Goss, 1987; Cresswell \& Kirkegaard, 1995), 从而使土壤的渗透性能和抗冲性 能得到提高。Gyssels 和 Poesen(2003) 研究了死根对 土壤可蚀性的影响, 发现随着死根生物量和死根长 度的增加, 细沟间可蚀性参数随之降低, 二者之间存 在着指数关系, 细沟间可蚀性与死根生物量和根系 长度之间的关系可以应用于水蚀预报模型 (Water erosion prediction project, WEPP) 等土壤侵蚀预报模 型中, 作为预报细沟间可蚀性的调整值。

在土壤抗冲性研究的过程中, 研究人员采用了 不同的方法对土壤抗冲性进行研究, 主要有静水崩 解法、原状土冲刷法、野外小区放水法及土壤理化性 质分析法等 4 种 (蒋定生等, 1995)。同时由于研究 方法不同，描述的指标也多种多样。其中原状坡面 冲刷法(周佩华和武春龙, 1993 ) 可以保证土壤不受 扰动, 且地形、汇流和土壤受力等都保持原有状况, 克服了冲刷槽的不足, 是一种更为科学的实验方法。 为了进一步深入系统地揭示草被植被根系对不同土 层深度上土壤侵蚀产沙特征的影响, 进而为揭示植 被覆盖条件下细沟侵蚀发育机理并对其进行预报， 本文首先对草被植被根系的垂直分布特征进行了深 入细致的调查, 在原状坡面冲刷法的基础上, 结合分
层处理, 对黄土地区草本植被覆盖下土壤垂直侵蚀 产沙规律进行了探讨。

\section{1 材料和方法}

\section{1 研究地区概况}

实验地点设在陕西省长武县西部洪家乡境内的 王东沟小流域, 此地处于黄土高原沟壑区中部, 海拔 $950 \sim 1225 \mathrm{~m}$, 年平均气温 $9.1{ }^{\circ} \mathrm{C}$, 年降水量为 $584.1 \mathrm{~mm}$, 年变率和月变率都较大, 最大次降雨量超 过 $80 \mathrm{~mm}$, 大部分都集中于 $7 \sim 9$ 月, 无霜期 $171 \mathrm{~d}$ 。 主要草地植被类型是以长芒草( Stipa bungeana)和白 羊草 (Bothriochlon ischaemum) 为优势种的多年生草 本群落, 其它伴生物种有胡枝子 (Lespedeza bicolor)、 野菊花( Dendranthema indicum) 等。

\section{2 分层冲刷试验小区的建立}

首先在坡面上建立大小为 $0.5 \mathrm{~m} \times 4 \mathrm{~m}$ 模拟冲 刷小区, 在表层冲刷实验进行完毕之后, 将表层 0 $5 \mathrm{~cm}$ 土壤去掉, 在相同流量下进行冲刷, 之后按照 分层冲刷的实验设计再将另外一层的土壤去掉, 在 下一个土壤深度上进行同样流量的冲刷实验。每两 次冲刷之间的时间间隔为 $12 \mathrm{~h}$, 如此反复进行, 直 到达到预定的土壤深度。

由于试验场地及其它客观条件的限制, 不可能 将所有流量的分层冲刷实验在不同坡度上的原始坡 面进行, 同时为了使试验结果更加具有代表性, 在不 同坡度上进行分层冲刷试验的时候, 选择了 10.5 和 $12.5 \mathrm{~L} \cdot \mathrm{min}^{-1}$ 作为放水流量进行冲刷实验, 具体冲 刷小区的分布及试验设计见表 1 。

表 1 分层冲刷实验小区的基本概况

Table 1 Brief introduction of runoff scouring plots

\begin{tabular}{|c|c|c|c|c|c|c|c|}
\hline $\begin{array}{l}\text { 小区编号 } \\
\text { Plot No. }\end{array}$ & $\begin{array}{c}\text { 流量 } \\
\text { Runoff discharge } \\
\left(\mathrm{L} \cdot \mathrm{min}^{-1}\right)\end{array}$ & $\begin{array}{c}\text { 坡度 } \\
\text { Slope } \\
\left({ }^{\circ}\right)\end{array}$ & $\begin{array}{c}\text { 植被覆盖度 } \\
\text { Vegetative } \\
\text { cover }\end{array}$ & $\begin{array}{c}\text { 土壤容重 } \\
\text { Bulk density } \\
\left(\mathrm{g} \cdot \mathrm{cm}^{-3}\right)\end{array}$ & $\begin{array}{c}\text { 坡向 } \\
\text { Direction }\end{array}$ & $\begin{array}{c}\text { 坡位 } \\
\text { Position }\end{array}$ & $\begin{array}{c}\text { 地点 } \\
\text { Location }\end{array}$ \\
\hline 1 & 6.5 & 20 & 0.86 & 1.28 & 南 South & 坡上 Upper & 黄白洼 Hangbaiwa \\
\hline 2 & 8.5 & 20 & 0.81 & 1.25 & 南 South & 坡上 Upper & 黄白洼 Hangbaiwa \\
\hline 3 & 10.5 & 20 & 0.82 & 1.23 & 南 South & 坡上 Upper & 黄白洼 Hangbaiwa \\
\hline 4 & 12.5 & 20 & 0.81 & 1.24 & 南 South & 坡上 Upper & 黄白洼 Hangbaiwa \\
\hline 5 & 14.5 & 20 & 0.85 & 1.27 & 南 South & 坡上 Upper & 黄白洼 Hangbaiwa \\
\hline 6 & 10.5 & 25 & 0.82 & 1.27 & 南 South & 坡中 Middle & 桐树洼 Tongshuwa \\
\hline 7 & 12.5 & 25 & 0.8 & 1.21 & 南 South & 坡中 Middle & 桐树洼 Tongshuwa \\
\hline 8 & 14.5 & 25 & 0.81 & 1.29 & 南 South & 坡中 Middle & 桐树洼 Tongshuwa \\
\hline 9 & 10.5 & 32 & 0.82 & 1.25 & 南 South & 坡中 Middle & 桐树洼 Tongshuwa \\
\hline 10 & 12.5 & 32 & 0.8 & 1.31 & 南 South & 坡中 Middle & 桐树洼 Tongshuwa \\
\hline 11 & 10.5 & 8 & 0.82 & 1.28 & 南 South & 坡下 Lower & 岳家山 Yuejiashan \\
\hline 12 & 12.5 & 8 & 0.82 & 1.24 & 南 South & 坡下 Lower & 岳家山 Yuejiashan \\
\hline
\end{tabular}




\section{3 根系调查}

在分层冲刷小区的两侧均匀布点 (图 1), 采用 土钻法 $(\varphi=9 \mathrm{~cm})$ 进行根系调查。然后用土钻在 $100 \mathrm{~cm}$ 的土壤剖面上, 以每 $10 \mathrm{~cm}$ 为一层进行采样, 用笁子将各层土壤进行过篮, 并拣出所有根系, 编号 后装入塑料袋带回实验室进行进一步的分析。将样 品带回实验室后, 用水将根系洗出, 晾干后装入信 封。对所获得的根系样品用蒸馏水清洗干净后置入 $105{ }^{\circ} \mathrm{C}$ 烘箱中, 烘干至恒重, 再分别称重和记录。

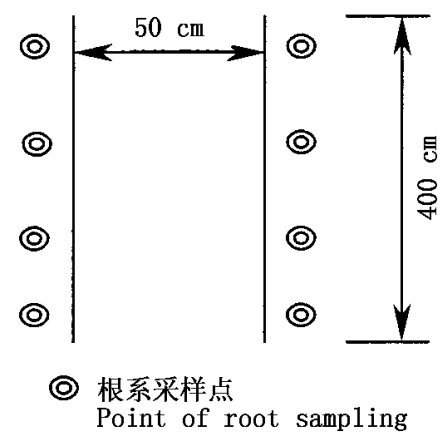

图 1 冲刷小区根系采样点示意图

Fig.1 Sketch map of root sampling on runoff scouring plot

\section{2 结果与讨论}

\section{1 不同地点根系生物量的垂直分布特征}

与乔木或者灌木等物种的根系相比, 草本植被 的根系比较细小, 并且根系的径级一般小于 $1 \mathrm{~mm}$ 。 结合前人的有关研究结果(李勇, 1995), 可以认为所 有草本植被的根系都能够起到有效网络土壤、提高 土壤抵抗径流的能力。因此在本研究中, 没有对草 本植被根系的径级进行进一步的划分, 而是直接应 用草本植被根系的全部生物量进行进一步的分析。

从植被根系生物量的分布特征可以看出 (图 2 ), 在 3 个立地上草本植被的根系分布特征具有基 本相似的规律, 各地点上草本植被的根系生物量都 表现出了明显的随着土壤深度增加而减少的趋势, 一般在土壤超过 $40 \mathrm{~cm}$ 的土层中、根系生物量的分 布达到一个相对稳定的数值, 说明在土壤深层, 草本 植被的根系分布较少。尽管在不同地点上草本植被 根系的垂直分布特征略有不同, 但是其分布特征相 似, 并且生物量相近; 采用根系扫描仪对根系长度、 表面积等形态指标的分析结果具有相似的变化规 律, 因此可以认为是一致的试验条件 (李鹏等, 2005)。

2.2 不同深度和坡度下坡面径流侵蚀产沙特征

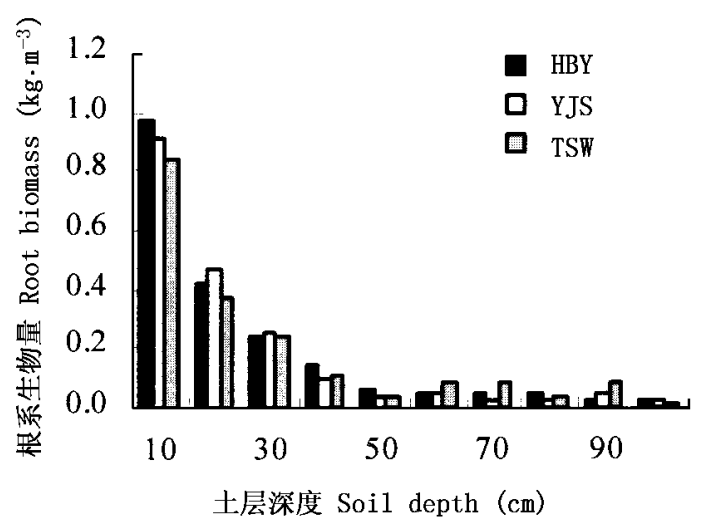

图 2 不同地点草本植被根系生物量分布特征

Fig.2 Vertical root biomass distribution of herbaceous on different sites HBY: 黄白洼 Huangbaiwa YJS: 岳家山 Yuejianshan TSW: 桐树 洼 Tongshuwa

\subsection{1 不同流量下土壤垂直侵蚀产沙特征}

在相同坡度下, 随着放水流量的逐渐增加, 坡面 上的侵蚀产沙量也相应增加。在表层土壤 $0 \sim 15 \mathrm{~cm}$ 的土壤剖面上,不同流量下径流侵蚀产沙量比较接 近, 不同深度上 $(0 \sim 5 \mathrm{~cm}$ 和 $10 \sim 15 \mathrm{~cm})$ 上进行冲刷 试验产沙量也比较接近。这说明植被根系的存在, 有效提高了表层土壤对径流侵蚀的抵抗能力, 根系 对径流侵蚀的影响是占主导地位的, 但是随着土壤 深度的增加, 不同深度上, 不同流量之间的侵蚀产沙 量产生了明显的差别, 一般在深度超过 $40 \mathrm{~cm}$ 时, 不 同深度上的侵蚀产沙量就发生了明显的变化。结合 植被根系的垂直分布特征可以看出, 在土壤深度超 过 $40 \mathrm{~cm}$ 后, 深层土壤中植被根系的分布数量较少, 存在的大部分根系是细小的毛细根, 对径流侵蚀的 抵抗能力较小。因此在土壤深度超过 $40 \mathrm{~cm}$ 后, 根 系对土壤侵蚀产沙的影响降低, 此时的侵蚀产沙量 主要与土壤本身的可蚀性和放水流量等有关。从表 2 中可以看出, 在土壤深度超过 $40 \mathrm{~cm}$ 后, 随着放水 流量的增加, 相同层次上的侵蚀产沙量也随之增加。

\subsection{2 不同坡度下土壤垂直侵蚀产沙特征}

一般来说径流流量越大, 侵蚀产沙量越高; 坡度 越大, 侵蚀产沙量也随之增高。从表 2 中可以看出, 不同流量和坡度下在不同土壤深度上进行的冲刷试 验具有相似的规律性。在土壤表层, 由于植被根系 的存在, 在不同坡度表层土壤上进行的分层冲刷试 验的侵蚀产沙量比较接近, 说明在表层土壤上, 根系 对侵蚀产沙的影响是占主导地位。在土壤深度超过 $15 \mathrm{~cm}$ 后, 随着坡度的增加, 对应深度上径流侵蚀产 沙量逐渐增加, 说明随着坡度的增加, 根系对土壤侵 
表 2 不同流量和坡度下不同土壤深度上侵蚀产沙量

Table 2 Sediment yield on different soil depth under different slope and runoff discharge

\begin{tabular}{|c|c|c|c|c|c|c|c|c|c|c|}
\hline \multirow{3}{*}{$\begin{array}{c}\text { 地点 } \\
\text { Site }\end{array}$} & \multirow{3}{*}{$\begin{array}{c}\text { 流量 Runoff } \\
\text { discharge } \\
\left(\mathrm{L} \cdot \mathrm{min}^{-1}\right)\end{array}$} & \multicolumn{9}{|c|}{ 深度 Depth $(\mathrm{cm})$} \\
\hline & & $0 \sim 5$ & $5 \sim 10$ & $10 \sim 15$ & $15 \sim 20$ & $20 \sim 25$ & $25 \sim 30$ & $30 \sim 40$ & $40 \sim 50$ & $50 \sim 60$ \\
\hline & & \multicolumn{9}{|c|}{ 产沙量 Sediment yield $\left(\mathrm{g} \bullet \mathrm{min}^{-1}\right)$} \\
\hline 岳家山( $\left.8^{\circ}\right)$ & 12.5 & 33.14 & 25.06 & 31.68 & - & 37.73 & 37.84 & 46.00 & 59.16 & - \\
\hline Yuejiashan & 10.5 & 11.27 & 20.81 & 23.87 & - & 34.73 & 34.05 & 38.90 & 50.84 & - \\
\hline 黄白洼 $\left(20^{\circ}\right)$ & 6.5 & 19.48 & 18.23 & 24.81 & 25.27 & 25.73 & 28.14 & 33.48 & 62.90 & - \\
\hline \multirow[t]{4}{*}{ Huangbaiwa } & 8.5 & 14.24 & 20.67 & 12.46 & 18.13 & 23.81 & 16.80 & 65.71 & 111.53 & - \\
\hline & 10.5 & 16.47 & 25.17 & 21.00 & 36.86 & 23.24 & 65.06 & 84.91 & 129.91 & 156.19 \\
\hline & 12.5 & 22.47 & 91.25 & 31.94 & 69.32 & 53.99 & 80.94 & 161.42 & 255.79 & - \\
\hline & 14.5 & 29.20 & 24.09 & 25.28 & 26.46 & 40.08 & 114.61 & 177.14 & 271.77 & - \\
\hline 桐树洼 $25^{\circ}$ & 10.5 & 15.91 & 19.57 & 22.50 & 39.67 & 111.83 & 118.42 & 134.43 & 143.03 & - \\
\hline \multirow[t]{2}{*}{ Tongshuwa } & 12.5 & 22.30 & 29.67 & 39.86 & 71.33 & 98.93 & 130.44 & 135.39 & 149.51 & - \\
\hline & 14.5 & 28.03 & 25.18 & 45.39 & 80.33 & 107.62 & 133.19 & 151.42 & 179.71 & - \\
\hline 桐树洼 32 & 10.5 & 14.99 & 20.67 & 31.67 & 118.20 & 143.64 & 168.41 & 250.03 & 285.75 & - \\
\hline Tongshuwa & 12.5 & 56.63 & 61.42 & 93.60 & 101.10 & 122.21 & 160.71 & 240.05 & 285.55 & - \\
\hline
\end{tabular}

- : 没有进行冲刷实验的层面 No scouring conducted on that depth

蚀产沙的影响逐渐降低。这说明, 尽管植被可以有 效的提高表层土壤抵抗径流侵蚀的能力。但是土壤 层次超过一定深度后, 植被根系对于土壤性质的改 善和提高的作用受到了一定程度的限制, 特别是在 土壤深度超过 $40 \mathrm{~cm}$ 后, 这种限制作用更加明显。

此外, 在试验过程中观察到随着土壤深度的不 断进行, 坡面上径流侵蚀的形态也在发生变化。在 土壤表层, 坡面上的侵蚀形态是以面蚀和片蚀的形 式进行, 随着土壤深度的加深, 在坡面上逐渐有跌坎 形成, 跌坎的大小和数量随着深度的增加而增加; 在 较大流量和较大坡度的情况下, 跌坎出现的深度变 浅, 也就是说在较浅的土壤深度上就会出现跌坎, 并 且在 $40 \sim 50 \mathrm{~cm}$ 的深度上这些跌坎逐渐连通, 有向 细沟侵蚀转化的趋势。同时在实验过程中也观测 到, 冲刷形成的跌坎中同样存在着毛细根, 说明并不 是所有的根系都能够有效的提高土壤抵抗径流冲刷 的能力。

\section{3 土壤垂直侵蚀产沙特征与根系生物量关系}

上述研究结果表明, 草地土壤侵蚀产沙的垂直 变化规律与根系密度的垂直分布特征密切相关, 根 系密度大, 侵蚀产沙少, 反之亦然。从图 3 中可以看 出, 随着土壤中根系含量的增加, 土壤垂直方向上侵 蚀产沙逐渐减少。采用曲线拟合的方法对土壤侵蚀 产沙与根系之间的关系进行模拟, 确定植被根系生 物量与垂直侵蚀产沙特征之间的关系拟合方程如 下:

$$
Y=\frac{1}{a+b \ln x}
$$

式中 $Y$ 为土壤侵蚀产沙的垂直特征; $x$ 是根系

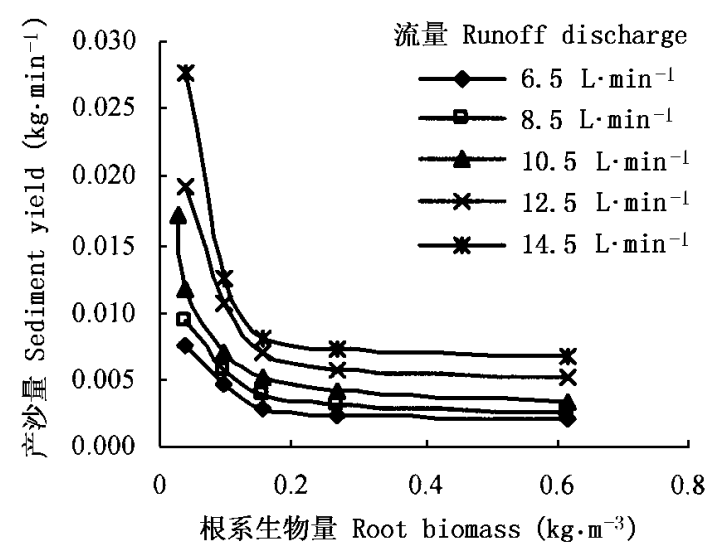

图 3 根系生物量与垂直侵蚀产沙量的关系

Fig.3 Relation between vertical sediment yield and root biomass

生物量, $a 、 b$ 为常数。

模拟分析结果表明(表 3), 公式较好地反映了 不同流量条件下根系特征与侵蚀产沙的关系, 在不 同流量条件下, 常数 $a 、 b$ 的值随着流量的增加而降 低,可能与该类土壤的可蚀性有关。

在有关植被根系的研究中, 研究人员对于描述 根系所应该采用的根系指标进行了探讨。在有关根 系生理、生态特征的研究中, 研究人员认为(李鹏等, 2005; 刘国涁, 1997), 采用根系的长度、表面积等指 标能够更加有效地反映根系与土壤的接触面积并反 映出根系在土壤中的生理作用。从植被与土壤侵蚀 的本质联系来说, 根系提高和改善土壤性质等方面 的作用主要是通过根系转成为土壤有机质, 进而改 善土壤团聚体等土壤性质而发挥作用的, 因此在研 究植被根系对土壤侵蚀影响的研究中, 采用根系生 
表 3 模拟方程中参数 $\boldsymbol{a} 、 \boldsymbol{b}$ 的确定

Table 3 Determination for the constant of $a$ and $b$ in the equation

\begin{tabular}{cccccc}
\hline 流量 Runoff discharge $\left(\mathrm{L} \cdot \mathrm{min}^{-1}\right)$ & 6.5 & 8.5 & 10.5 & 12.5 & 14.5 \\
\hline$a$ & 547.8126 & 411.9401 & 338.4305 & 226.8418 & 200.5907 \\
$b$ & 125.8726 & 92.8135 & 78.6954 & 53.0848 & 49.8790 \\
标准差 $S D$ & 0.0005 & 0.0005 & 0.0006 & 0.0008 & 0.0009 \\
相关系数 Correlative coefficient $(R)$ & 0.9817 & 0.9893 & 0.9956 & 0.9933 & 0.9958 \\
\hline
\end{tabular}

物量更能够从本质上反映植被根系与土壤有机质之 间的关系, 是一个更加合理的指标。此外,在分层冲 刷的过程中观察到冲刷形成的跌坎中有毛细根存 在, 这说明并不是所有的细根都能够有效地提高土 壤抵抗侵蚀的性能, 只有径级在一定范围之内的细 根有效。采用根系生物量来描述植被根系与土壤抵 抗侵蚀性能之间的关系既考虑了毛细根的作用, 又 弱化了其它指标(如根系长度、根系表面积等)带来 的影响, 是一个合理的指标。

\section{3 结 论}

本文通过对黄土地区天然草地土壤垂直侵蚀产 沙规律及其与根系分布特征关系的研究, 可以得到 以下结论:

1)不同流量和坡度下进行的分层冲刷试验结果 表明, 在土壤表层, 不同坡度、流量下侵蚀产沙量之 间没有明显的差异, 说明在土壤表层植被根系对侵 蚀产沙的影响是占主导地位的。但是在土层超过一 定深度后, 根系的分布数量减少, 不同流量和坡度下 的侵蚀产沙发生了明显的变化, 不同深度上的侵蚀 量, 主要与坡度、流量及土壤本身的状况有关, 根系 提高土壤抵抗径流侵蚀产沙的能力受到了限制。

2) 随着土层深度的不断加大, 坡面上径流侵蚀 的形态也在发生变化, 又逐渐从面蚀向细沟侵蚀发 展的趋势。在实验过程中也观测到, 冲刷形成的跌 坎中同样存在着毛细根, 说明并不是所有的根系都 能够有效地提高土壤抵抗径流冲刷的能力, 太细的 根和太粗的根都不能发挥最佳的抗冲刷功效。

3) 草地土壤侵蚀产沙的垂直变化规律与根系密 度的垂直分布特征密切相关, 根系密度大, 侵蚀产沙 少, 反之亦然。根据试验观测结果, 采用曲线拟和的 方法对土壤侵蚀产沙与根系之间的关系进行模拟， 建立了植被根系生物量与垂直侵蚀产沙特征之间的 定量关系。

\section{参 考 文 献}

Cresswell HP, Kirkegaard JA (1995). Subsoil amelioration by plant roots the process and evident. Australian Journal of Soil Research, 33, $221-239$

Ghidey F, Alberts EE (1997). Plant root effects on soil erodibility, splash detachment, soil strength, and aggregate stability. Transaction of the ASAE, 40(1), 129 - 135.

Gyssels G, Poesen J (2003). The importance of plant root characteristics in controlling concentrated flow erosion rates. Earth Surface Processes and Landforms, 28, $371-384$.

Jiang DS (蒋定生), Fan XK (范兴科), Li XH (李新华) (1995). Researches on vertical and horizontal patterns of soil anti-scourablity on Loess Plateau. Journal of Soil and Water Conservation (水土保持学报), 9(2), 1-8. (in Chinese with English abstract)

Li P(李鹏), Li ZB(李占斌), Zhan TZ(澹台湛) (2005). Dynamics root distribution characters of herbaceous vegetation on the abandoned lands on the Loess Plateau. Journal of Applied Ecolo$g y$ (应用生态学报), 16, 849-853. (in Chinese with English abstract)

Li Y (李勇), Zhu XM (朱显谟), Tian JY (田积芗) (1991). Effectiveness of plant roots to increase the anti-scourability of soil on the Loess Plateau. Chinese Science Bulletin (科学通报), 12, 935 - 938. (in Chinese)

Li Y (李勇) (1995). Plant Root System and its Relation with Soil Anti-scourability (黄土高原植物根系与土壤抗冲性). Science Press, Beijing. (in Chinese)

Liu GB (刘国彬), Jiang DS (蒋定生), Zhu XM (朱显谟) (1996). Study on grass root bio-mechanics in Loess Plateau. Journal of Soil Erosion and Soil and Water Conservation (土壤侵 蚀与水土保持学报), 2(3), 21-28. (in Chinese with English abstract)

Liu GB (刘国涁) (1997). Soil anti-scourability research and its perspective in Loess Plateau. Research on Soil and Water Conservation (水土保持研究), 4(5), 91 - 101. (in Chinese with English abstract)

Reid BJ, Goss MJ (1987). Effect of living roots of different plant species on the aggregate stability of two arable soils. Journal of Soil Science, 32, $521-541$.

Waldron LJ, Dakessian S (1981). Soil reinforcement by roots: calculation of increased soil shear resistance from root properties. Soil Science, 132, $427-435$.

Zhou PH (周佩华), Wu CL (武春龙) (1993). Researches on the methods of soil anti-scourability on Loess Plateau. Journal of Soil and Water Conservation (水土保持学报), 7(1), 29-34. (in Chinese with English abstract)

Zhu XM (朱显谟), Tian JY (田积芗) (1993). Research on intensifying soil infiltration and soil anti-scourability on the Loess Plateau. Journal of Soil and Water Conservation (水土保持学 报), 7(3), 1-10. (in Chinese with English abstract) 